\title{
Microfluidic-Based Platforms for Biological Assays Automation in Life-Sciences Research and Remote Medical Applications
}

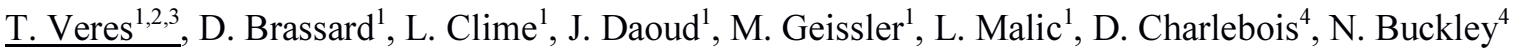 \\ ${ }^{1}$ National Research Council of Canada, 75 de Mortagne Boulevard, Boucherville, Québec, J4B 6 Y4 Canada. \\ ${ }^{2}$ Adjunct Professor, INRS- Énergie Matériaux Télécommunications \\ ${ }^{3}$ Biomedical Engineering, McGill University, 3775 University Street, Montréal, Québec, H3A 2B4 Canada. \\ ${ }^{4}$ Canadian Space Agency, 6767 route de l'Aéroport Saint-Hubert, Québec, J3Y $8 Y 9$ Canada.
}

The capacity to separate and analyse critical cellular and sub-cellular targets from complex matrices such as bodily fluids is required for emerging precision medicine approaches. Such approaches are important not only to empower emerging research in precision medicine in the translation of the molecular information in clinical care particularly challenging in remote applications.

Fueled by their research and clinical diagnostics importance, chip-based methods for isolation and on-site analysis of blood components, such as plasma, red blood cells, white blood cells, extracellular vesicles (EV, e.g., exosomes, microvesicles), proteins, and circulating nucleic acids (cfDNA, miRNA) are developing rapidly. These challenges are compounded by the need to process diverse samples (whole blood, saliva, urine, cell cultures, water and food) across a large set of volumes (from $\mu \mathrm{l}$ to few $\mathrm{ml}$ ). The rapid, on-site, automated preparation from body fluids and molecular analysis of circulation biomarkers that have scientific and clinical value is critically important for timely decision making process and has not been addressed so far. This is particularly important for remote applications including low-resources communities or space research and medical support for long term space missions.

Microfluidics and lab-on-chip (LOC) technologies have been developed over the last decade driven by the need for automation of complex analytical procedures in many clinical, industrial or research applications. LOC technologies can simplify human manipulations, reduce associated risks of contamination, decrease assay times, improve analytical throughput, and decrease reagent consumption. They also enable minimally trained personnel to perform analytical procedures outside laboratory settings. Several approaches for the manipulation of liquids have been developed including external pressure mediated pumping, electrowetting, centrifugal and capillary forces. Centrifugal microfluidic technologies stand out as a promising platform for sample preparation and integration of sample-to-answer assays able to handle samples ranging from $1 \mu \mathrm{l}$ to few $\mathrm{ml}$ with almost no dead volume. For space applications, this type of microfluidic platform is particularly appealing as the presence of a strong on-chip centrifugal acceleration, not only offers unique sample preparation capabilities (sedimentation of debris, density fractionation, etc.), but also makes the entire microfluidic process independent of normal gravity.

We have developed a novel centrifugal microfluidic platform, "Power-Blade", combining regulated pressure control in a centrifugal microfluidic platform as a mean to facilitate integration of complex assays in centrifugal microfluidic and improve reliability of fluidic manipulations. In this method (Fig. 1(a)), a programmable air pump and multiple miniature electromechanical valves are placed on a rotating stage and connected to microfluidic devices. The valves, pump and other active elements can be computer-controlled in real time while the platform is rotating at high speed to trigger fluidic displacements inside the microfluidic devices. Centrifugal fluidic actuation becomes independent of liquid-solid contact angles, the nature of the samples, and the chip fabrication materials, making this method very suitable for the manipulation of complex samples in space applications.

The Power-Blade platform's exceptional capabilities to automate complex biological samples preparation and integrate biomolecular assays has been demonstrated in a number of diverse applications. These applications include extraction of genomic DNA from cell culture lysates (L. Clime et al., Lab Chip. 2015, 15, p.2400) and rapid (1h) sample-to-answer VTEC E. coli bacteria detection and sub-typing with a very simple $5 \times 10 \mathrm{~cm}$ cartridge fabricated in low-cost thermoplastic (Fig.1 (c)). More recently, we have demonstrated automated extraction and purification of multiple target proteins and total genomic DNA from large volumes of whole blood. Figure 2(b) demonstrates that high efficiencies for protein extraction from blood samples can be achieved across the entire physiological range of interest. Three target protein biomarkers, TNF- $\alpha$, PTH and ALP, that encompass the whole physiological concentration range of interest for proteins biomarkers were tested (TNF- $\alpha$, low 0.3 to $1.3 \mathrm{pg} / \mathrm{mL}$; PTH, average 10 to $70 \mathrm{pg} / \mathrm{mL}$; ALP, high 10 to $70 \mathrm{ng} / \mathrm{mL}$ ). 
Figure 3(c) shows a summary of the extraction efficiency obtained for an average of 3 runs performed on each of 5 different blood donors $(n=3$ per donor). The results confirm that we can achieve very high extraction efficiencies ( $\sim 80 \%$ on average) with relatively low variance. The average extraction efficiency obtained for the manual assays is significantly lower $(\sim 5 \%)$ and exhibits much higher test-to-test variation.

We have also demonstrated automated workflow taking less than $1 \mathrm{~h}$ for total nucleic acids extraction from large samples whole blood using P-Blade operated microfluidic cartridges. As shown in Figure $3(b, c)$, the microfluidic protocol outperforms the most widely used commercially available NA extraction kits by providing significantly higher extraction yields and high eluted sample purity. The NA extraction yield, $\mathrm{A} 260 / 280$ and $\mathrm{A} 260 / 230$ ratios are also shown.

Figure 1: a) Schematic representation of the "Power Blade" centrifugal microfluidics principles; b) Laboratory version of the "Power Blade" hardware having PCR cycling built-in capability (tested in parabolic flights conditions); and c) Power-Blade operated polymer microfluidic device $(5 \times 10 \mathrm{~cm})$ performing bacterial lysis, on-chip DNA amplification implementing a molecular assay for VTEC E.Coli molecular sub-typing.
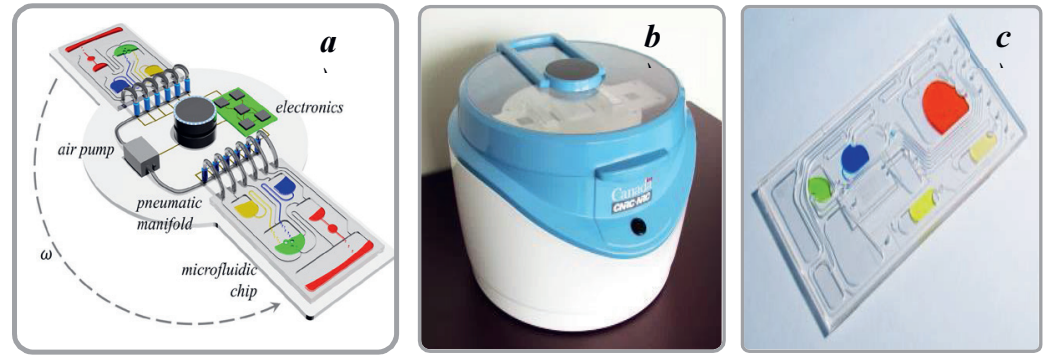

Figure 2: a) $5 \times 10 \mathrm{~cm}$ three layers thermoplastic polymer device operated on the power-Blade used for automated (45 min) extraction of multiple proteins from $1 \mathrm{ml}$ whole blood; b) Comparison of protein extraction efficiency for a test performed with the automated microfluidic cartridge (P-MicroPREP) and test performed manually; and c) Boxplot showing a summary of the protein extraction efficiency measured for all experiments performed with a manual procedure and with the automated
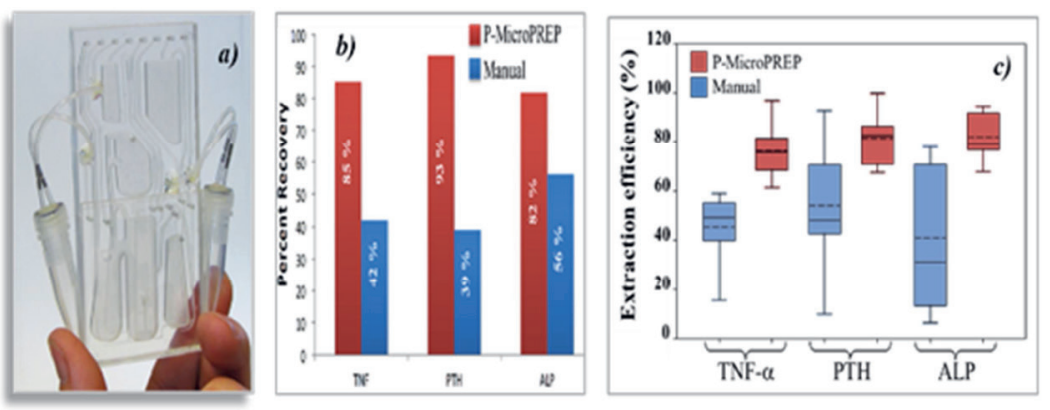
microfluidic cartridge.

Figure 3: a) Image of the $5 \times 10 \mathrm{~cm}$ device performing automated and rapid $(60 \mathrm{~min})$ total nucleic extraction (NA) from $1 \mathrm{ml}$ whole blood; b) Measured total DNA extraction yield from whole blood samples for multiple experiments performed on four commercial nucleic acids extraction kits and the performances of the microfluidic extraction on the same samples; and c) Spectra showing the absorbance of eluted $N A$ solution from whole blood using the microfluidic cartridges and various NA exaction commercial kits.
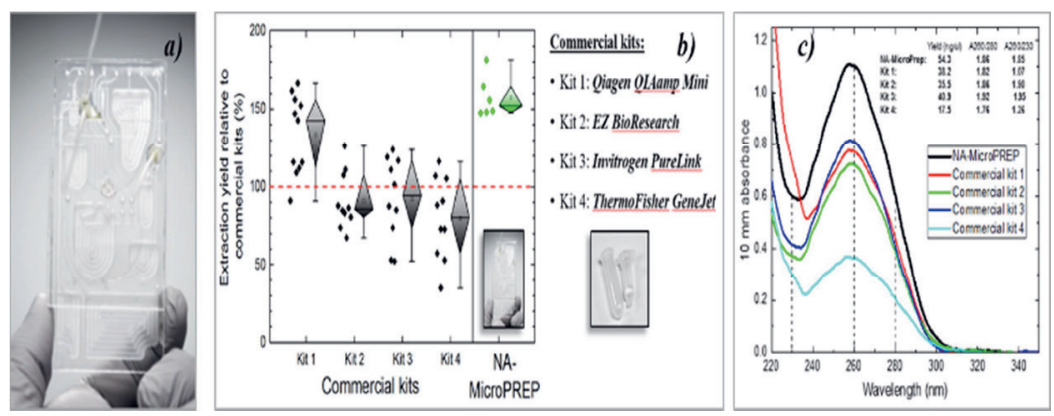

In this presentation we will discuss various applications developed around microfluidic technologies will ranging from (i) isolation and purification of cellular and circulating markers (proteins, nucleic acids, cfDNA, miRNA, exosomes) from complex matrices such as whole blood to, (ii) sample-to-answer detection of foodborne pathogens. These demonstrations highlight the potential of advanced lab-on-chip systems for biological sample preparation and assay automation and for deployment of complex assays outside normal laboratory settings particularly in remote life sciences research and medical diagnostics applications. 